\title{
Tri-meson-mixing of $\pi-\eta-\eta^{\prime}$ and $\rho-\omega-\phi$ in the light-cone quark model
}

\author{
Wen Qian and Bo-Qiang Ma \\ School of Physics and State Key Laboratory of Nuclear Physics and Technology, Peking University, Beijing 100871, China
}

Received: date / Revised version: date

\begin{abstract}
The radiative transition form factors of the pseudoscalar mesons $\pi, \eta, \eta^{\prime}$ and the vector mesons $\rho, \omega, \phi$ are restudied with $\pi-\eta-\eta^{\prime}$ and $\rho-\omega-\phi$ in tri-meson-mixing pattern, which is described by tri-mixing matrices in the light-cone constituent quark model. The experimental transition decay widths are better reproduced with tri-meson-mixing than previous results in a two-mixing-angle scenario of only two-meson $\eta-\eta^{\prime}$ mixing and $\omega-\phi$ mixing.
\end{abstract}

PACS. 1 2.39.Ki, 13.40.Gp, 14.40.-n, 14.40.Aq

\section{Introduction}

Light hadrons are well classified in SU(3) flavor multiplets [1] based on Gell-Mann [2] and Ne'eman [3] theory. The Gell-Mann-Okubo mass formula 2, 4, which exhibits the mass relation of hadrons, has a simple linear form and a more complicated quadratic form. In order to explain the deviation of the meson masses from the Gell-Mann-Okubo mass formula, two-meson $\omega-\phi$ mixing and $\eta-\eta^{\prime}$ mixing were introduced since the 1960s [5]. The mixing between mesons helps to understand the $\mathrm{SU}(3)$ symmetry breaking.

The departure from the $\omega$ - $\phi$ meson ideal mixing was studied [6] according to the meson mass spectrum, radiative decays and $2 \pi, 3 \pi$ decay modes which are related to isospin conservation and Okubo-Zweig-Iizuka (OZI) rule violation. It is involved in the study of $\tau$ decays for a decade years [7. The mixing of $\eta-\eta^{\prime}$ has also been studied based on pseudoscalar meson masses and their decays 8 9 10. However, recently more analysis focus on their decay constants $11,12,13$, and a mixing scheme were promoted in which two mixing angles were introduced. The $\eta-\eta^{\prime}$ mixing is also related to the study of $J / \psi$ decay [14].

In fact, the isospin violating mixing of $\rho-\omega$ and $\pi-\eta$ have been considered for a long time. $\rho-\omega$ mixing was first promoted by Glashow [15] based on electromagnetic interaction. This mixing plays an important role in the nuclear charge asymmetry study [16], and is related to the $\mathrm{CP}$ violation analysis in hadronic B decays [17, 18, 19] and charmed hadron decays $[20$. The off-shell behavior of $\rho-\omega$, $\pi-\eta$ mixing was also studied [21,22.

Then it is natural to consider the tri-meson $\pi-\eta-\eta^{\prime}$ mixing and $\rho-\omega-\phi$ mixing. Some efforts have been devoted to them respectively $[21,23,24,25,26$.

Many different methods were used in above studies of meson mixing, e.g., chiral perturbation theory, quantum chromodynamics(QCD) sum rule, nonrelativistic and rela- tivistic quark model, hidden local symmetry (HLS) model, et al. The light-cone constituent quark model [27, 28, 29] is also an important model that has been used to study the mixing of mesons [30,31,32,33,34. As far as properties of mesons are studied at low energy scales, nonperturbative QCD effects are important. The light-cone constituent quark model is a convenient model for incorporating the nonperturbative QCD effects effectively. With the Melosh-Wigner rotation [35, 36, 37, the SU(6) instant form wave function can be transformed into the light-front form to include relativistic effects. Taking the minimal quark-antiquark Fock state description of photons and mesons, we can calculate their transition form factors, decay widths and radii.

In the light-cone quark model, the mixing of pseudoscalar $\eta-\eta^{\prime}$ mesons has been studied using octet-singlet mixing scheme [38,39] and quark flavor scheme 40, 41, and recently together with the vector meson $\omega$ - $\phi$ mixing, it is studied with a two-mixing-angle scenario 42, which can reproduce data better phenomenologically. When introducing two mixing angles, the mixing matrices are nonunitary. Concerning the isospin symmetry breaking, in this paper we restudy the transition form factors between vector mesons and pseudoscalar mesons with tri-meson $\rho-\omega-\phi$ mixing and $\pi-\eta-\eta^{\prime}$ mixing in the light-cone quark model, where the mixing is described by unitary tri-mixing matrices 23, 43. This can also be seen as an explanation of the non-unitary mixing matrices in the two-mixing-angle scenario 42 .

Since in this paper all the $I_{z}=0$ mesons are involved in mixing, all the parameters have to be readjusted together without priority. With the experimental data of the meson radii, decay widths as constraints, we reset all the parameters in our model. New results for the pion form factor between $Q^{2}=0.6$ and $2.45 \mathrm{GeV}^{2}$ at the Thomas 
Jefferson National Accelerator Facility (JLab) were presented recently [44. We recalculate the $Q^{2}$ behavior of the pseudo scalar meson form factors in our model using the new parameters and compare them with the latest experimental data. The $Q^{2}$ behavior of the transition form factors of other pseudoscalar and vector mesons are also recalculated, and extrapolated to the time-like region and compared with experimental data 45, 46, 47, 48.

This paper is organized as following. In Sec. 2 we explain the tri-meson-mixing of $\pi-\eta-\eta^{\prime}$ and $\rho-\omega-\phi$ respectively. In Sec. 3, the definitions of decay constants, form factors and radiative transition form factors and their calculation in the light-cone constituent quark model are briefly reviewed. In Sec. 4, we reset the parameters of our model by using the experimental data as constraints. The $Q^{2}$ behaviors of form factors are compared with experimental data.

\section{Tri-meson-mixing of $\rho-\omega-\phi$ and $\pi-\eta-\eta^{\prime}$}

The $\eta-\eta^{\prime}$ and $\omega-\phi$ mixing were studied based on different theories. Different values of mixing angles were determined by different ways in literatures: the pseudoscalar meson $\eta-\eta^{\prime}$ mixing angle is around $20^{\circ}$ and the vector meson $\omega$ $\phi$ mixing is around $28^{\circ} \sim 44^{\circ}$, based on the Gell-MannOkubo mass formula, two-photon decay widths, radiative and strong decay widths and so on 49 .

Considering the discussion of pseudoscalar meson mixing in the chiral perturbation theory [12,11, 40, and PCAC formulas of flavour singlet current [50], the following mixing form

$$
\begin{aligned}
\left(\begin{array}{c}
|\eta\rangle \\
\left|\eta^{\prime}\right\rangle
\end{array}\right) & =\left(\begin{array}{cc}
\cos \theta_{q s}^{S} & -\sin \theta_{q s}^{S} \\
\sin \theta_{q s}^{S} & \cos \theta_{q s}^{S}
\end{array}\right)\left(\begin{array}{l}
\left|\eta_{q}\right\rangle \\
\left|\eta_{s}\right\rangle
\end{array}\right), \\
\left(\begin{array}{cc}
f_{\eta}^{q} & f_{\eta}^{s} \\
f_{\eta^{\prime}}^{q} & f_{\eta^{\prime}}^{s}
\end{array}\right) & =\left(\begin{array}{cc}
\cos \theta_{q s}^{S} & -\sin \theta_{q s}^{S} \\
\sin \theta_{q s}^{S} & \cos \theta_{q s}^{S}
\end{array}\right)\left(\begin{array}{cc}
f_{q} & 0 \\
0 & f_{s}
\end{array}\right),
\end{aligned}
$$

with only one mixing angle is not enough to describe meson mixing.

So in the light-cone constituent quark model phenomenology studying, the meson mixing of $\eta-\eta^{\prime}$ and $\omega-\phi$ with two mixing angles are expressed as following forms [42]:

$$
\begin{aligned}
& \left(\begin{array}{l}
|\eta\rangle \\
\left|\eta^{\prime}\right\rangle
\end{array}\right)=\left(\begin{array}{cc}
\cos \theta_{q}^{S} & -\sin \theta_{s}^{S} \\
\sin \theta_{q}^{S} & \cos \theta_{s}^{S}
\end{array}\right)\left(\begin{array}{l}
\left|\eta_{q}\right\rangle \\
\left|\eta_{s}\right\rangle
\end{array}\right), \\
& \left(\begin{array}{l}
|\phi\rangle \\
|\omega\rangle
\end{array}\right)=\left(\begin{array}{cc}
\cos \theta_{q}^{V} & -\sin \theta_{s}^{V} \\
\sin \theta_{q}^{V} & \cos \theta_{s}^{V}
\end{array}\right)\left(\begin{array}{l}
\left|\omega_{q}\right\rangle \\
\left|\omega_{s}\right\rangle
\end{array}\right)
\end{aligned}
$$

where, the quark-flavour basis mixing scheme is denoted by the subscripts $q s$, with quark flavor bases (for $\psi=\eta$ or $\omega)$ 33, 41

$$
\begin{aligned}
\left|\psi_{q}\right\rangle & =\frac{1}{\sqrt{2}}(u \bar{u}+d \bar{d}) \varphi^{q}\left(x, \mathbf{k}_{\perp}\right), \\
\left|\psi_{s}\right\rangle & =s \bar{s} \varphi^{s}\left(x, \mathbf{k}_{\perp}\right) .
\end{aligned}
$$

In the octet-singlet scheme (denoted as 08) 38, 39, just replace $\left\{\theta_{q s}^{S, V}, \eta_{q, s}, \omega_{q, s}\right\}$ in above formula with $\left\{\theta_{08}^{S, V}, \eta_{0,8}\right.$, $\left.\omega_{0,8}\right\}$. Here, the flavor $\mathrm{SU}(3)$ octet basis and the singlet basis are (for $\psi=\eta$ or $\omega$ ).

$$
\begin{aligned}
& \left|\psi_{8}\right\rangle=\frac{1}{\sqrt{6}}(u \bar{u}+d \bar{d}) \varphi_{8}^{q}\left(x, \mathbf{k}_{\perp}\right)-\frac{2}{\sqrt{6}} s \bar{s} \varphi_{8}^{s}\left(x, \mathbf{k}_{\perp}\right) \\
& \left|\psi_{0}\right\rangle=\frac{1}{\sqrt{3}}(u \bar{u}+d \bar{d}) \varphi_{0}^{q}\left(x, \mathbf{k}_{\perp}\right)+\frac{1}{\sqrt{3}} s \bar{s} \varphi_{0}^{s}\left(x, \mathbf{k}_{\perp}\right)
\end{aligned}
$$

$\theta_{08}^{S, V}$ and $\theta_{q s}^{S, V}$ are respectively pseudoscalar and vector meson mixing angles in the octet-singlet scheme and the quark flavor scheme.

Meanwhile, the tri-mixing of pseudoscalar mesons $\pi$ $\eta-\eta^{\prime}$ and vector mesons $\rho-\omega-\phi$ have been discussed respectively with different forms and parametrization [26, 21, 23, 24,25]. Confined in a unitary mixing of the ideal basis, in this paper we accept the tri-mixing matrix [23] for describing the meson mixing, as

$$
\left(\begin{array}{c}
\omega \\
\rho \\
\phi
\end{array}\right)=M V\left(\begin{array}{c}
\omega_{I} \\
\rho_{I} \\
\phi_{I}
\end{array}\right),\left(\begin{array}{c}
\pi \\
\eta \\
\eta^{\prime}
\end{array}\right)=M S\left(\begin{array}{c}
\pi_{I} \\
\eta_{q} \\
\eta_{s}
\end{array}\right)
$$

where,

$$
\begin{aligned}
\rho_{I} & =\frac{1}{\sqrt{2}}(u \bar{u}-d \bar{d}) \varphi_{\rho_{I}}, & \pi_{I} & =\frac{1}{\sqrt{2}}(u \bar{u}-d \bar{d}) \varphi_{\pi_{I}}, \\
\omega_{I} & =\frac{1}{\sqrt{2}}(u \bar{u}+d \bar{d}) \varphi_{\omega_{I}}, & \eta_{q} & =\frac{1}{\sqrt{2}}(u \bar{u}+d \bar{d}) \varphi_{\eta_{q}} \\
\phi_{I} & =-s \bar{s} \varphi_{\phi_{I}}, & \eta_{s} & =s \bar{s} \varphi_{\eta_{s}}
\end{aligned}
$$

$M V=\left(\begin{array}{ccc}\cos \delta_{V} \cos \beta_{V} & -\sin \delta_{V} \cos \beta_{V} & \sin \beta_{V} \\ \sin \delta_{V} \cos \gamma_{V}+\cos \delta_{V} \sin \beta_{V} \sin \gamma_{V} \cos \delta_{V} \cos \gamma_{V}-\sin \delta_{V} \sin \beta_{V} \sin \gamma_{V} & -\cos \beta_{V} \sin \gamma_{V} \\ \sin \delta_{V} \sin \gamma_{V}-\cos \delta_{V} \sin \beta_{V} \cos \gamma_{V} \cos \delta_{V} \sin \gamma_{V}+\sin \delta_{V} \sin \beta_{V} \cos \gamma_{V} & \cos \beta_{V} \cos \gamma_{V}\end{array}\right)$.

$M V$ is the vector meson tri-mixing matrix. $M S$ is the pseudoscalar meson tri-mixing matrix and it has the same structure of $M V$ with $\left\{\delta_{V}, \beta_{V}, \gamma_{V}\right\}$ replaced by $\left\{\delta_{S}, \beta_{S}, \gamma_{S}\right\}$, that is, both the pseudoscalar mixing and vector mixing are described by three mixing angles. Since we do not discuss the $\mathrm{CP}$ violation and complex phases, the mixing angles are all real in this paper. The wave function $\varphi_{i}=A_{i} \exp \left[-\frac{m_{u}^{2}+\mathbf{k}_{\perp}^{2}}{8 \beta_{i}^{2} x(1-x)}\right]$, for $i=\omega_{I}, \rho_{I}, \pi_{I}, \eta_{q} ; \varphi_{i}=$ $A_{i} \exp \left[-\frac{m_{s}^{2}+\mathbf{k}_{\perp}^{2}}{8 \beta_{i}^{2} x(1-x)}\right]$, for $i=\phi_{I}, \eta_{s}$, where we adopt the Brodsky-Huang-Lepage (BHL) prescription [28,51.

Thus, the decay constants and transition form factors can be calculated by the following tri-mixing formulas,

$$
\left(\begin{array}{l}
F_{\pi \rightarrow \gamma \gamma^{*}}\left(Q^{2}\right) \\
F_{\eta \rightarrow \gamma \gamma^{*}}\left(Q^{2}\right) \\
F_{\eta^{\prime} \rightarrow \gamma \gamma^{*}}\left(Q^{2}\right)
\end{array}\right)=M S\left(\begin{array}{l}
F_{\pi_{I} \rightarrow \gamma \gamma^{*}}\left(Q^{2}\right) \\
F_{\eta_{q} \rightarrow \gamma \gamma^{*}}\left(Q^{2}\right) \\
F_{\eta_{s} \rightarrow \gamma \gamma^{*}}\left(Q^{2}\right)
\end{array}\right)
$$

$$
\left(\begin{array}{l}
f_{\omega} \\
f_{\rho} \\
f_{\phi}
\end{array}\right)=M V\left(\begin{array}{l}
f_{\omega_{I}} \\
f_{\rho_{I}} \\
f_{\phi_{I}}
\end{array}\right)
$$




$$
\left(\begin{array}{l}
F_{\omega \rightarrow \pi \gamma^{*}}\left(Q^{2}\right) \\
F_{\omega \rightarrow \eta \gamma^{*}}\left(Q^{2}\right) \\
F_{\eta^{\prime} \rightarrow \omega \gamma^{*}}\left(Q^{2}\right) \\
F_{\rho \rightarrow \pi \gamma^{*}}\left(Q^{2}\right) \\
F_{\rho \rightarrow \eta \gamma^{*}}\left(Q^{2}\right) \\
F_{\eta^{\prime} \rightarrow \rho \gamma^{*}}\left(Q^{2}\right) \\
F_{\phi \rightarrow \pi \gamma^{*}}\left(Q^{2}\right) \\
F_{\phi \rightarrow \eta \gamma^{*}}\left(Q^{2}\right) \\
F_{\phi \rightarrow \eta^{\prime} \gamma^{*}}\left(Q^{2}\right)
\end{array}\right)=(M V \otimes M S)\left(\begin{array}{l}
F_{\omega_{I} \rightarrow \pi_{I} \gamma^{*}}\left(Q^{2}\right) \\
F_{\omega_{I} \rightarrow \eta_{q} \gamma^{*}}\left(Q^{2}\right) \\
0 \\
F_{\rho_{I} \rightarrow \pi_{I} \gamma^{*}}\left(Q^{2}\right) \\
F_{\rho_{I} \rightarrow \eta_{I} \gamma^{*}}\left(Q^{2}\right) \\
0 \\
0 \\
0 \\
F_{\phi_{I} \rightarrow \eta_{I} \gamma^{*}}\left(Q^{2}\right)
\end{array}\right) .
$$

\section{Decay constants and radiative transition form factors in the light-cone quark model}

In the $P \rightarrow \mu \nu$ process, the decay constant $f_{P}$ of the charged pseudoscalar meson $P$ is defined as,

$$
\left\langle 0\left|J^{\mu}\right| P(p)\right\rangle=i \sqrt{2} f_{P} p^{\mu},
$$

where, $J^{\mu}$ is the axial-vector part of the charged weak current. The charged form factor $F_{P}\left(Q^{2}\right)$ of a pseudoscalar meson $P$ is

$$
\left\langle P\left(p^{\prime}\right)\left|J^{\mu}\right| P(p)\right\rangle \delta^{3}\left(\mathbf{p}-\mathbf{p}^{\prime}+\mathbf{q}\right)=F_{P}\left(Q^{2}\right)\left(p+p^{\prime}\right)^{\mu},
$$

where, $J^{\mu}=e \bar{\psi} \gamma^{\mu} \psi, Q^{2}=-q^{2}$; and the electromagnetic radius of the pseudoscalar meson is

$$
\left\langle r_{P}^{2}\right\rangle=-\left.6 \frac{\partial F_{P}\left(Q^{2}\right)}{\partial Q^{2}}\right|_{Q^{2}=0} .
$$

The transition form factor of $P \rightarrow \gamma \gamma^{*}$ is defined by $[52$ 53.

$$
\begin{aligned}
& \left\langle\gamma(p-q)\left|J^{\mu}\right| P(p, \lambda)\right\rangle \\
= & i e^{2} F_{P \rightarrow \gamma \gamma^{*}}\left(Q^{2}\right) \varepsilon^{\mu \nu \rho \sigma} p_{\nu} \epsilon_{\rho}(p-q, \lambda) q_{\sigma},
\end{aligned}
$$

so the decay width of $P \rightarrow \gamma \gamma$ is

$$
\Gamma(P \rightarrow \gamma \gamma)=\frac{1}{4} \pi \alpha^{2} M_{P}^{3}\left|F_{P \rightarrow \gamma \gamma^{*}}(0)\right|^{2} .
$$

In the $V \rightarrow e^{+} e^{-}$process, the decay constant of the vector meson $V$ is defined by,

$$
\left\langle 0\left|j_{\mu}\right| V\left(p, S_{z}\right)\right\rangle=M_{V} f_{V} \epsilon_{\mu}\left(S_{z}\right) .
$$

Then, the decay width of $V \rightarrow e^{+} e^{-}$is

$$
\Gamma\left(V \rightarrow e^{+} e^{-}\right)=\frac{4 \pi \alpha^{2} f_{V}^{2}}{3 M_{V}} .
$$

The radiative transition form factor between a pseudoscalar meson $P$ and a vector meson $V$ is defined by [53]

$$
\begin{aligned}
& \left\langle P\left(p^{\prime}\right)\left|J^{\mu}\right| V(p, \lambda)\right\rangle \\
= & i e F_{V \rightarrow P \gamma}\left(Q^{2}\right) \varepsilon^{\mu \nu \rho \sigma} \epsilon_{\nu}(p, \lambda) p_{\rho}^{\prime} p_{\sigma},
\end{aligned}
$$

where, $\epsilon(p, \lambda)$ is the polarization vector of the vector meson. Thus the decay widths of $V \rightarrow P \gamma$ and $P \rightarrow V \gamma$ are

$$
\begin{aligned}
& \Gamma_{V \rightarrow P \gamma}=\frac{\alpha}{3}\left|F_{V \rightarrow P \gamma^{*}}(0)\right|^{2}\left(\frac{M_{V}^{2}-M_{P}^{2}}{2 M_{V}}\right)^{3}, \\
& \Gamma_{P \rightarrow V \gamma}=\alpha\left|F_{P \rightarrow V \gamma^{*}}(0)\right|^{2}\left(\frac{M_{P}^{2}-M_{V}^{2}}{2 M_{P}}\right)^{3} .
\end{aligned}
$$

To calculate the above decay constants and transition form factors of pseudoscalar and vector mesons, we use the light-cone quark model with the lowest Fock state expansion as an approximation. The wave function of a meson is simplified to be

$$
\begin{aligned}
& \left|M\left(P^{+}, \mathbf{P}_{\perp}, S_{z}\right)\right\rangle \\
= & \sum_{\lambda_{i}} \int \prod_{i=1}^{2} \frac{\mathrm{d} x_{i} \mathrm{~d}^{2} \mathbf{k}_{\perp i}}{\sqrt{x_{i}} 16 \pi^{3}} 16 \pi^{3} \delta\left(1-\sum_{i=1}^{2} x_{i}\right) \delta^{(2)}\left(\sum_{i=1}^{2} \mathbf{k}_{\perp i}\right) \\
& \times\left|n: x_{i} P^{+}, x_{i} \mathbf{P}_{\perp}+\mathbf{k}_{\perp i}, \lambda_{i}\right\rangle \psi_{2 / M}\left(x_{i}, \mathbf{k}_{\perp i}, \lambda_{i}\right) \\
= & \int \frac{\mathrm{d} x \mathrm{~d}^{2} \mathbf{k}_{\perp}}{\sqrt{x(1-x)} 16 \pi^{3}} \varphi\left(x, \mathbf{k}_{\perp}\right) \chi_{M}^{S_{z}}\left(x, \mathbf{k}_{\perp}, \lambda_{1}, \lambda_{2}\right),
\end{aligned}
$$

where, the momentum-space wave function $\varphi\left(x, \mathbf{k}_{\perp}\right)$ is described by the BHL prescription 28,51]

$$
\begin{aligned}
\varphi\left(x, \mathbf{k}_{\perp}\right) & =\varphi_{\mathrm{BHL}}\left(x, \mathbf{k}_{\perp}\right) \\
& =A \exp \left[-\frac{1}{8 \beta^{2}}\left(\frac{m_{1}^{2}+\mathbf{k}_{\perp}^{2}}{x}+\frac{m_{2}^{2}+\mathbf{k}_{\perp}^{2}}{1-x}\right)\right](26)
\end{aligned}
$$

and $\chi_{M}^{S_{z}}\left(x, \mathbf{k}_{\perp}, \lambda_{1}, \lambda_{2}\right)$ is the spin wave function.

Through the Melosh-Wigner rotation [36, 35, 37.

$$
\left\{\begin{array}{l}
\chi_{i}^{\uparrow}(T)=w_{i}\left[\left(k_{i}^{+}+m_{i}\right) \chi_{i}^{\uparrow}(F)-k_{i}^{R} \chi_{i}^{\downarrow}(F)\right] \\
\chi_{i}^{\downarrow}(T)=w_{i}\left[\left(k_{i}^{+}+m_{i}\right) \chi_{i}^{\downarrow}(F)+k_{i}^{L} \chi_{i}^{\uparrow}(F)\right]
\end{array},\right.
$$

where $w_{i}=1 / \sqrt{2 k_{i}^{+}\left(k^{0}+m_{i}\right)}, k^{R, L}=k^{1} \pm k^{2}, k^{+}=k^{0}+$ $k^{3}=x \mathcal{M}, \mathcal{M}=\sqrt{\frac{\mathbf{k}_{\perp}^{2}+m_{1}^{2}}{x}+\frac{\mathbf{k}_{\perp}{ }^{2}+m_{2}^{2}}{1-x}}$, or corresponding proper meson vertex $[53,55]$,

$$
\bar{u}\left(k_{1}, \lambda_{1}\right) \Gamma_{M} v\left(k_{2}, \lambda_{2}\right),
$$

with

$$
\begin{aligned}
\Gamma_{P}= & \frac{1}{\sqrt{2} \sqrt{\mathcal{M}^{2}-\left(m_{1}-m_{2}\right)^{2}}} \gamma_{5}, \text { for pseudoscalar mesons } \\
\Gamma_{V}= & -\frac{1}{\sqrt{2} \sqrt{\mathcal{M}^{2}-\left(m_{1}-m_{2}\right)^{2}}}\left(\gamma^{\mu}-\frac{k_{1}^{\mu}-k_{2}^{\mu}}{\mathcal{M}+m_{1}+m_{2}}\right) \\
& \times \epsilon_{\mu}\left(P, S_{z}\right), \text { for vector mesons, }
\end{aligned}
$$

we get the light-cone spin wave function of mesons and calculate the above decay constants and transition form factors. The formulas were listed in the appendix of Ref. 42 . 


\section{Numerical Calculation Results}

In the light-cone constituent quark model, when only one mixing angle is introduced to describe the pseudoscalar meson mixing, the angle can actually be analytically determined by the $Q^{2} \rightarrow \infty$ limit behavior of $F_{P \gamma \gamma^{*}}\left(Q^{2}\right)$ [41,33. However, when two or three mixing angles are introduced, there is no more analytic solution. So in this paper, the meson mixing angles and all the parameters in wavefunction are determined phenomenologically by electroweak properties of the mesons by fitting light-cone constituent quark model results to experimental data.

In this paper, all parameters including $m_{u}, m_{s}$, wave function parameters of $\pi, K, \eta_{q}, \eta_{s}, \rho_{I}, \omega_{I}, \phi_{I}$ are set together by using the experimental data, which include electromagnetic decay widths and radii. We assume that the wave function parameters of $\pi^{ \pm}$are the same as those of $\pi_{I}$. Though the off-shell behavior of $\pi-\eta-\eta^{\prime}$ has been discussed in some papers, we assume the mixing angles to be constants when $Q^{2}$ changing. The $Q^{2}$ behavior of the radiative transition form factors are recalculated using the reset parameters and compared with experimental data. The input data are all from PDG08 [54, as listed in Table 1.

The $Q^{2} \rightarrow \infty$ limiting behavior of $Q^{2} F_{P \rightarrow \gamma \gamma^{*}}$ is also considered as constraints for setting the parameters [27, 41]:

$$
\lim _{Q^{2} \rightarrow \infty} Q^{2} F_{P \rightarrow \gamma \gamma^{*}}\left(Q^{2}\right)=2 c_{P} f_{P}=\frac{2 c_{P}^{2}}{4 \pi^{2} F_{P \rightarrow \gamma \gamma^{*}}(0)},(30)
$$

where,

$$
c_{P}=\left(c_{\pi_{I}}, c_{\eta_{q}}, c_{\eta_{s}}\right)=\left(1, \frac{5}{3}, \frac{\sqrt{2}}{3}\right) .
$$

The results are shown in Table 1, together with the results of Ref. 42. As we can see, the reproduction of the radiative decay widths are improved with tri-mesonmixing pattern. In fact, since $\beta_{S}, \delta_{S} \sim 0$, the mixing matrix $M S$ is similar to the mixing of $\eta, \eta^{\prime}$, and in the fit result $\gamma_{S}=39.40^{\circ}$ is compatible with the results we got in the two-mixing angle scenario of the quark flavor scheme $\theta_{q}^{S}=40.57^{\circ}, \theta_{s}^{S}=43.89^{\circ}$. The vector meson mixing bases are a little different from the ones in the two-mixing angle scenario of the quark flavor scheme, which is equivalent to a $90^{\circ}$ rotation: $90^{\circ}-\theta_{q}^{V}=3.29^{\circ}, 90^{\circ}-\theta_{s}^{V}=-3.43^{\circ}$. The $\beta_{V}=-6.2^{\circ}$ got in the tri-meson-mixing fit is also compatible with them.

There were following form relations between the mixing angles introduced in Ref. [40],

$$
\begin{gathered}
\theta_{8}=\phi-\arctan \frac{\sqrt{2} f_{s}}{f_{q}} \\
\theta_{0}=\phi-\arctan \frac{\sqrt{2} f_{q}}{f_{s}} .
\end{gathered}
$$

When three mixing angles are introduced, relations are not so simple. Similar to derivation in Ref. 40,

$$
\left(\begin{array}{ccc}
f_{\pi}^{\pi} & f_{\pi}^{q} & f_{\pi}^{s} \\
f_{\eta}^{\pi} & f_{\eta}^{q} & f_{\eta}^{s} \\
f_{\eta^{\prime}}^{\pi} & f_{\eta^{\prime}}^{q} & f_{\eta^{\prime}}^{s}
\end{array}\right)=M S\left(\delta_{S}, \beta_{S}, \gamma_{S}\right)\left(\begin{array}{ccc}
f_{\pi} & 0 & 0 \\
0 & f_{q} & 0 \\
0 & 0 & f_{s}
\end{array}\right)
$$

$$
\left(\begin{array}{ccc}
f_{\pi}^{\pi} & f_{\pi}^{8} & f_{\pi}^{0} \\
f_{\eta}^{\pi} & f_{\eta}^{8} & f_{\eta}^{0} \\
f_{\eta^{\prime}}^{\pi} & f_{\eta^{\prime}}^{8} & f_{\eta^{\prime}}^{0}
\end{array}\right)=M S\left(\delta_{S}, \beta_{S}, \gamma_{S}\right)\left(\begin{array}{ccc}
f_{\pi} & 0 & 0 \\
0 & f_{q} & 0 \\
0 & 0 & f_{s}
\end{array}\right) M S^{\dagger}\left(0,0, \gamma_{\text {ideal }}\right)
$$

where, $\gamma_{\text {ideal }}=\arctan \sqrt{2} .\left\{f_{\pi}^{8}, f_{\pi}^{0}, f_{\eta}^{\pi}, f_{\eta^{\prime}}^{\pi}\right\}$ are very small, if they are ignored, two mixing schemes can be related by

$$
\left(\begin{array}{ccc}
f_{\pi}^{\pi} & f_{\pi}^{8} & f_{\pi}^{0} \\
f_{\eta}^{\pi} & f_{\eta}^{8} & f_{\eta}^{0} \\
f_{\eta^{\prime}}^{\pi} & f_{\eta^{\prime}}^{8} & f_{\eta^{\prime}}^{0}
\end{array}\right) \sim\left(\begin{array}{ccc}
f_{\pi}^{\pi} & 0 & 0 \\
0 & f_{\eta}^{8} & f_{\eta}^{0} \\
0 & f_{\eta^{\prime}}^{8} & f_{\eta^{\prime}}^{0}
\end{array}\right)=\left(\begin{array}{ccc}
f_{\pi}^{\pi} & 0 & 0 \\
0 & f_{8} \cos \theta_{8}^{S} & -f_{0} \sin \theta_{0}^{S} \\
0 & f_{8} \sin \theta_{8}^{S} & f_{0} \cos \theta_{0}^{S}
\end{array}\right),
$$

that leads to

$$
\begin{aligned}
\tan \theta_{8}^{S} & =\frac{\cos \delta_{S} \tan \gamma_{S}+\sin \beta_{S} \sin \delta_{S}-\sqrt{2} \frac{f_{S}}{f_{q}} \cos \beta_{S}}{\cos \delta_{S}-\sin \beta_{S} \tan \gamma_{S} \sin \delta_{S}+\sqrt{2} \frac{f_{S}}{f_{q}} \cos \beta_{S} \tan \gamma_{S}} \\
\tan \theta_{0}^{S} & =\frac{\cos \beta_{S} \tan \gamma_{S}-\sqrt{2} \frac{f_{q}}{f_{S}}\left(\cos \delta_{S}-\sin \beta_{S} \sin \delta_{S} \tan \gamma_{S}\right)}{\cos \beta_{S}-\sqrt{2} \frac{f_{q}}{f_{S}}\left(\cos \delta_{S} \tan \gamma_{S}-\sin \beta_{S} \sin \delta_{S}\right)} .
\end{aligned}
$$

Here, the $f_{\pi}^{\pi}, f_{q}, f_{s}$ are respectively equal to $\frac{1}{4 \pi^{2} F_{\psi \gamma \gamma^{*}}(0)}$ (for $\left.\psi=\pi_{I}, \eta_{q}, \eta_{s}\right)$.

With the parameters set in this paper, we have

$$
\begin{aligned}
& f_{\pi}^{\pi}=0.091 \mathrm{GeV}, \\
& f_{q}=0.097 \mathrm{GeV}, \\
& f_{s}=0.194 \mathrm{GeV} .
\end{aligned}
$$

Putting the $\beta_{S}, \delta_{S}, \gamma_{S}$ shown in the fifth column of Table1 into Eqs. (36), one can get $\theta_{8}^{S}=-30.96^{\circ}$, and $\theta_{0}^{S}=3.93^{\circ}$. These two values are close to the results we got when using the two-mixing angle scheme as shown in the third column of Table 1

The $Q^{2}$ behavior of meson form factors is recalculated, as shown in Figs. 1,4, In comparison with experimental data, the $Q^{2}$ behavior fit data well in the low $Q^{2}$ region. Extrapolating $Q^{2}$ to the time-like region by $q_{\perp} \rightarrow i q_{\perp}$ [56], we also recalculate the form factors in the limited timelike region. The results are comparable with experimental data, as shown in Fig. 5 and Fig. 6.

In our calculation, only the relation got in the $Q^{2} \rightarrow \infty$ limit is used to determine parameters, and perturbative QCD effect is not actually taken into account when the form factors are calculated. So, in fact the $Q^{2} F_{P \gamma \gamma^{*}}\left(Q^{2}\right)$ and $Q^{2} F_{P}\left(Q^{2}\right)$ calculated in our paper should only be valid when $Q^{2}$ ranges from 0 to around a few $\mathrm{GeV}^{2}$.

\section{Conclusion}

In this paper we use the light-cone constituent quark model to calculate the properties of the pseudoscalar mesons $\pi$ $\eta-\eta^{\prime}$ and the vector mesons $\rho-\omega-\phi$ with tri-meson-mixing pattern, which is described by unitary tri-mixing matrices. All parameters including $m_{u}, m_{s}$, the wave function parameters of $\pi, K, \eta_{q}, \eta_{s}, \rho_{I}, \omega_{I}, \phi_{I}$ are constrained together by the experimental data, including their electromagnetic decay widths and radii. The reproduction of the experimental decay widths turn out to be better than 
Table 1. Experimental data [54 for the decay constants and decay widths of $\eta, \omega, \phi$ are compared with theoretical values. The first column is the experimental data. The second and third column are the theoretical results of two-mixing angle scenario [42]. The fourth column is the theoretical result with tri-meson-mixing pattern. Parameters set in different schemes are listed below.

\begin{tabular}{|c|c|c|c|c|}
\hline & $F_{\exp } / f_{\exp }(\mathrm{GeV})$ & $\begin{array}{c}F_{\mathrm{th}} / f_{\mathrm{th}}(\mathrm{GeV}) \\
\text { (two-angle } \\
08 \text { scheme })\end{array}$ & $\begin{array}{c}F_{\mathrm{th}} / f_{\mathrm{th}}(\mathrm{GeV}) \\
\text { (two-angle } \\
\text { qs scheme) }\end{array}$ & $\begin{array}{c}F_{\mathrm{th}} / f_{\mathrm{th}}(\mathrm{GeV}) \\
\text { (tri-meson-mixing) }\end{array}$ \\
\hline$f_{\pi^{+}}$ & $0.0922 \pm 0.0001$ & 0.0922 & 0.0922 & 0.0920 \\
\hline$\left\langle r_{\pi}^{2}\right\rangle \mathrm{fm}^{2}$ & $0.45 \pm 0.01$ & 0.45 & 0.45 & 0.45 \\
\hline$F_{\pi^{0} \rightarrow \gamma \gamma^{*}}(0)$ & $0.274 \pm 0.010$ & 0.274 & 0.274 & 0.279 \\
\hline$f_{K^{+}}\left(\vec{K}^{+} \rightarrow \mu \nu\right)$ & $0.1100 \pm 0.0006$ & 0.1100 & 0.1100 & 0.1106 \\
\hline$\left\langle r_{K^{+}}^{2}\right\rangle \mathrm{fm}^{2}$ & $0.31 \pm 0.03$ & 0.31 & 0.31 & 0.31 \\
\hline$\left\langle r_{K^{0}}^{2}\right\rangle \mathrm{fm}^{2}$ & $-0.077 \pm 0.010$ & -0.077 & -0.077 & -0.077 \\
\hline$F_{\eta \rightarrow \gamma \gamma^{*}}(0)$ & $0.272 \pm 0.007$ & 0.272 & 0.259 & 0.277 \\
\hline$F_{\eta^{\prime} \rightarrow \gamma \gamma^{*}}(0)$ & $0.342 \pm 0.006$ & 0.342 & 0.317 & 0.334 \\
\hline$f_{\rho}\left(\rho \rightarrow e^{+} e^{-}\right)$ & $0.1564 \pm 0.0007$ & 0.1564 & 0.1564 & 0.1603 \\
\hline$f_{\phi}\left(\phi \rightarrow e^{+} e^{-}\right)$ & $0.076 \pm 0.012$ & 0.068 & 0.076 & 0.075 \\
\hline$f_{\omega}\left(\omega \rightarrow e^{+} e^{-}\right)$ & $0.0459 \pm 0.0008$ & 0.0475 & 0.0456 & 0.04556 \\
\hline$F_{\rho+\rightarrow \pi^{+\gamma}}(0)$ & $0.83 \pm 0.06$ & 0.83 & 0.83 & 0.84 \\
\hline$F_{\phi \rightarrow \pi \gamma^{*}}(0)$ & $0.133 \pm 0.003$ & 0.131 & 0.132 & 0.132 \\
\hline$F_{\omega \rightarrow \pi \gamma^{*}}(0)$ & $2.385 \pm 0.004$ & 2.327 & 2.295 & 2.382 \\
\hline$F_{\phi \rightarrow \eta \gamma^{*}}(0)$ & $-0.692 \pm 0.007$ & -0.581 & -0.662 & -0.677 \\
\hline$F_{\phi \rightarrow \eta^{\prime} \gamma^{*}}(0)$ & $0.712 \pm 0.01$ & 0.853 & 0.742 & 0.727 \\
\hline$F_{\omega \rightarrow \eta \gamma^{*}}(0)$ & $0.449 \pm 0.02$ & 0.453 & 0.457 & 0.454 \\
\hline$F_{\eta^{\prime} \rightarrow \omega \gamma^{*}}(0)$ & $0.460 \pm 0.03$ & 0.450 & 0.470 & 0.461 \\
\hline$F_{\eta \rightarrow \gamma \gamma^{*}}(0)$ & $0.272 \pm 0.007$ & 0.272 & 0.259 & 0.277 \\
\hline$F_{\eta^{\prime} \rightarrow \gamma \gamma^{*}}(0)$ & $0.342 \pm 0.006$ & 0.342 & 0.317 & 0.334 \\
\hline$F_{\rho \rightarrow \eta \gamma^{*}}(0)$ & $1.59 \pm 0.05$ & 1.59 & 1.66 & 1.50 \\
\hline$F_{\eta^{\prime} \rightarrow \rho \gamma^{*}}(0)$ & $1.35 \pm 0.06$ & 1.35 & 1.42 & 1.39 \\
\hline$\theta^{V}$ & & $\begin{array}{l}\theta_{8}^{V}=12.17^{\circ} \\
\theta_{0}^{V}=77.82^{\circ}\end{array}$ & $\begin{array}{l}\theta_{q}^{V}=86.71^{\circ} \\
\theta_{s}^{V}=93.43^{\circ}\end{array}$ & $\begin{array}{c}\beta_{V}=-6.20^{\circ} \\
\delta_{V}=1.40^{\circ} \\
\gamma_{V}=-3.70^{\circ}\end{array}$ \\
\hline$\theta^{S}$ & & $\begin{array}{c}\theta_{8}^{S}=-26.18^{\circ} \\
\theta_{0}^{S}=-2.85^{\circ}\end{array}$ & $\begin{array}{l}\theta_{q}^{S}=40.57^{\circ} \\
\theta_{s}^{S}=43.89^{\circ}\end{array}$ & $\begin{array}{c}\beta_{S}=-4.20^{\circ} \\
\delta_{S}=-1.41^{\circ} \\
\gamma_{S}=39.40^{\circ}\end{array}$ \\
\hline $\begin{array}{c}\text { Parameters } \\
A\left(\mathrm{GeV}^{-1}\right) \\
\beta(\mathrm{GeV})\end{array}$ & & $\begin{array}{c}m_{u}=0.198 \\
m_{s}=0.556 \\
A_{K}=68.73 \\
\beta_{K}=0.405 \\
A_{\pi}=47.36 \\
\beta_{\pi}=0.411 \\
A_{\eta 8}=41.65 \\
\beta_{\eta 8}=0.607 \\
A_{\eta 0}=32.12 \\
\beta_{\eta 0}=0.925 \\
A_{\rho}=48.585 \\
\beta_{\rho}=0.373 \\
A_{\omega 8}=215.18 \\
\beta_{\omega 8}=0.332 \\
A_{\omega 0}=135.52 \\
\beta_{\eta 0}=0.358\end{array}$ & $\begin{array}{l}m_{u}=0.198 \\
m_{s}=0.556 \\
A_{K}=68.73 \\
\beta_{K}=0.405 \\
A_{\pi}=47.36 \\
\beta_{\pi}=0.411 \\
A_{\eta q}=34.40 \\
\beta_{\eta q}=0.525 \\
A_{\eta s}=91.39 \\
\beta_{\eta s}=0.525 \\
A_{\rho}=48.585 \\
\beta_{\rho}=0.373 \\
A_{\omega q}=51.58 \\
\beta_{\omega q}=0.330 \\
A_{\omega s}=52.28 \\
\beta_{\omega s}=0.490\end{array}$ & $\begin{aligned} m_{u} & =0.198 \\
m_{s} & =0.556 \\
A_{K} & =68.54 \\
\beta_{K} & =0.407 \\
A_{\pi_{I}} & =47.36 \\
\beta_{\pi_{I}} & =0.410 \\
A_{\eta q} & =38.79 \\
\beta_{\eta q} & =0.486 \\
A_{\eta s} & =95.46 \\
\beta_{\eta s} & =0.486 \\
A_{\rho_{I}} & =38.14 \\
\beta_{\rho_{I}} & =0.411 \\
A_{\omega_{I}} & =41.45 \\
\beta_{\omega_{I}} & =0.419 \\
A_{\phi_{I}} & =63.16 \\
\beta_{\phi_{I}} & =0.476\end{aligned}$ \\
\hline$=\sum\left(\frac{F_{\mathrm{th}}-F_{\exp }}{F_{\exp }}\right)^{2}$ & & 0.0786 & 0.0259 & 0.0083 \\
\hline
\end{tabular}




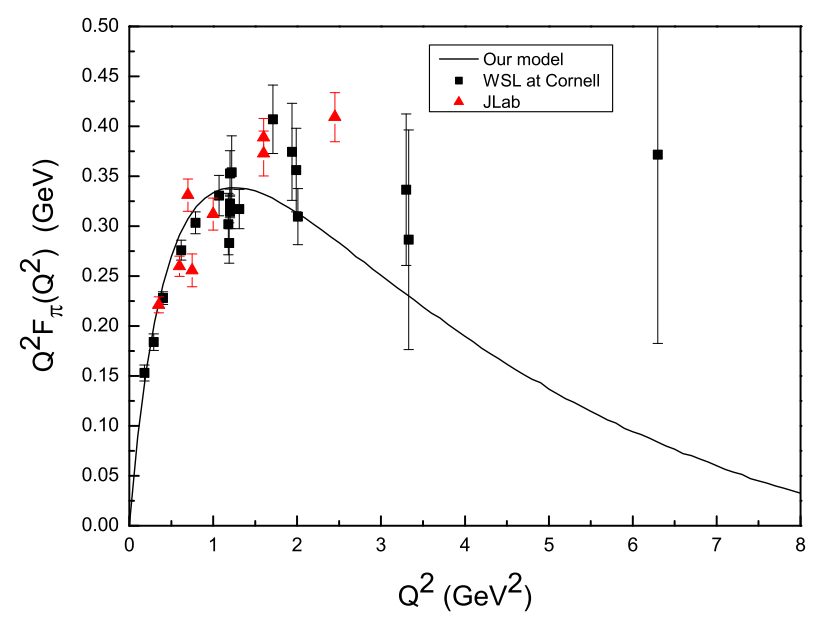

Fig. 1. The $Q^{2}$ behavior of the form factor $Q^{2} F_{\pi}\left(Q^{2}\right)$ compared with experimental data 45,44. The solid squares are data measured by the Wilson Synchrotron Laboratory at Cornell University. The solid triangles are data given by JLab.

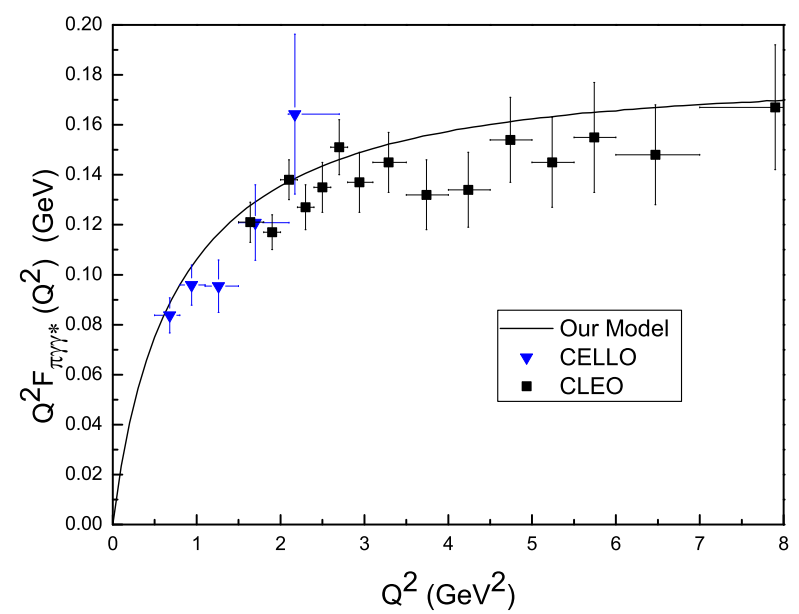

Fig. 2. The $Q^{2}$ behavior of the form factor $Q^{2} F_{\pi \rightarrow \gamma \gamma^{*}}\left(Q^{2}\right)$ compared with experimental data [46, 47.

only two-meson mixing of $\eta-\eta^{\prime}$ and $\omega$ - $\phi$ were introduced, as can be seen from $\chi$ 's in Table 11. We recalculate the $Q^{2}$ behavior of the radiative transition form factors, and extrapolate them to the limited time-like region. Results are comparable with experimental data. The tri-mesonmixing pattern in this paper can explain why the twomixing-angle scenario with non-unitary mixing matrices of two mesons could give acceptable results in our previous work 42 .

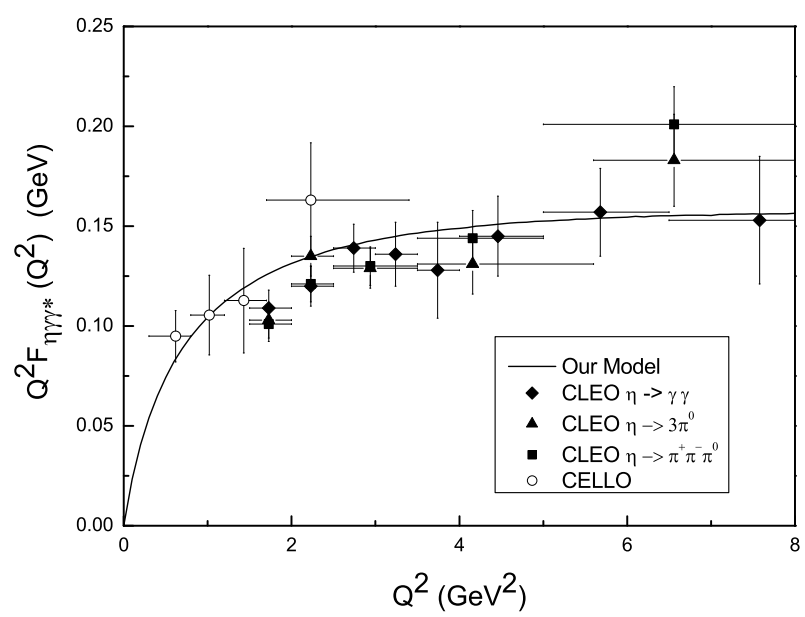

Fig. 3. The $Q^{2}$ behavior of the form factor $Q^{2} F_{\eta \rightarrow \gamma \gamma^{*}}\left(Q^{2}\right)$ compared with experimental data [46,47.

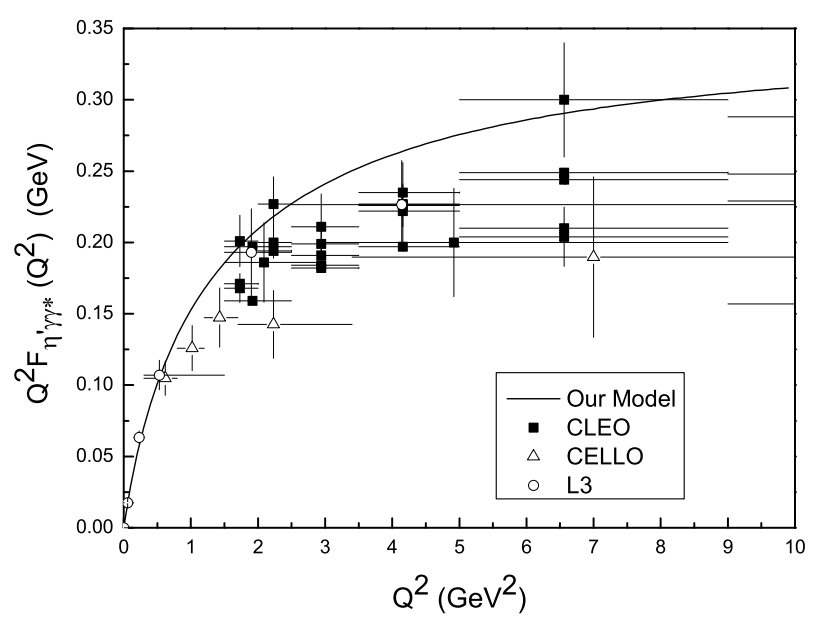

Fig. 4. The $Q^{2}$ behavior of the form factor $Q^{2} F_{\eta^{\prime} \rightarrow \gamma \gamma^{*}}\left(Q^{2}\right)$ compared with experimental data 46, 47,48.

\section{Acknowledgment}

This work is partially supported by National Natural Science Foundation of China (No. 10721063 and No. 10975003) and by the Key Grant Project of Chinese Ministry of Education (No. 305001).

\section{References}

1. M. Gell-Mann, Phys. Lett. 8, (1964) 214; G. Zweig, An SU(3) Model (1964).

2. M. Gell-Mann, The eightfold way, W. A. Benjamin, NY (1961).

3. Y. Ne'eman, Nucl. Phys. 26,(1961) 222. 


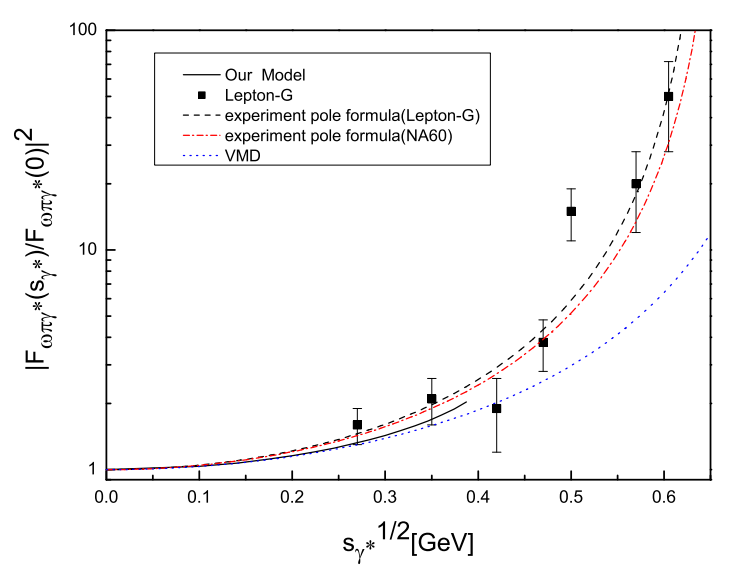

Fig. 5. The $Q^{2}$ behavior of the form factor $Q^{2} F_{\omega \rightarrow \pi \gamma^{*}}\left(Q^{2}\right)$ compared with experimental data. The dashed-dotted line is the result of fitting NA60 data with the pole dependence [57. and the dashed line is that of fitting Lepton G data [58. The dotted line is the vector meson dominance (VMD) model prediction.

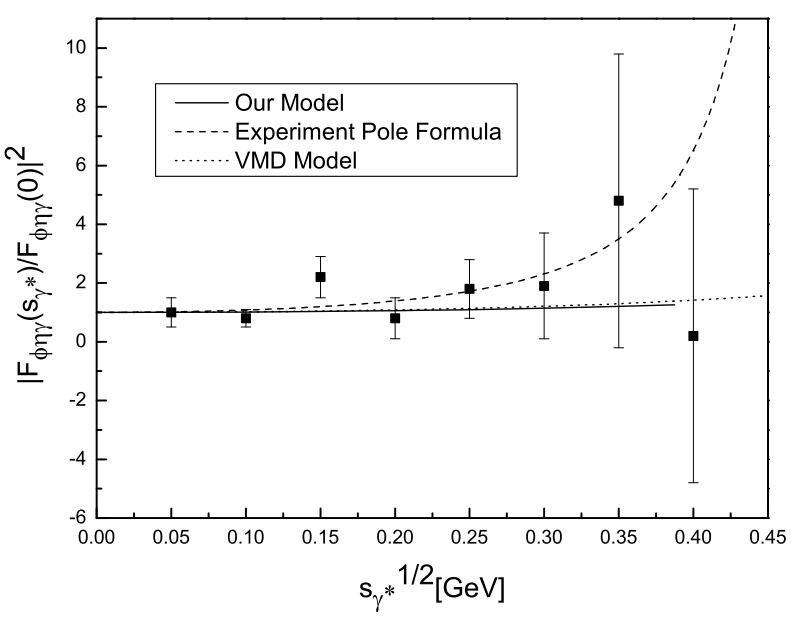

Fig. 6. The $Q^{2}$ behavior of the form factor $Q^{2} F_{\phi \rightarrow \eta \gamma^{*}}\left(Q^{2}\right)$ compared with experimental data $[59$. The dashed-dotted line is the result of fitting experimental data with the pole dependence. The dotted line is the VMD model prediction.

4. M. Gell-Mann, Phys. Rev. 125, (1962) 1067; S. Okubo, Progr. Theoret. Phys. 27, (1962) 949.

5. J. J. Sakurai, Phys. Rev. Lett. 9, (1962) 472 ; S. Coleman, Phys. Rev. 135, (1964) B779.

6. S. Okubo, Phys. Lett. 5,(1963) 165; J. Harte and G. Sachs, Phys. Rev. 135, (1964) B459; L. Epele, H. Fanchiotti and A. G. Grunfeld, Eur. Phys. J. C 30,(2004) 97; A. Kucukarslan and Ulf-G. Meissner, Mod. Phys. Lett. A 21, (2006) 1423.

7. G. Lopez Castro and D.A. Lopez Falcon, Phys. Rev. D 54,(1996) 4400; K.R. Nasriddinov, B.N. Kuranov, G.G. Takhtamyshev and T. A. Merkulova, Phys. Atom. Nucl. 64,
(2001) 1326, Yad. Fiz. 64, (2001) 1402; M. Benayoun, P. David, L. Del Buono, O. Leitner and H.B. O'Connell, Nucl. Phys. Proc. Suppl. 181, (2008) 161.

8. N. Isgur, Phys. Rev. D 13, (1976) 122.

9. H. Fritzsch and J.D. Jackson, Phys. Lett. B 66, (1977) 365. 10. K. Kawarabayashi and N. Ohta, Nucl. Phys. B175, (1980) 477; Prog. Theor. Phys. 66, (1981) 1789.

11. H. Leutwyler, Nucl. Phys. Proc. Suppl. 64, (1998) 223.

12. R. Kaiser and H. Leutwyler, arXiv:hep-ph/9806336

13. T. Feldmann and P. Kroll, Eur. Phys. J. C 5, (1998) 327. For earlier treatments of two mixing angles for the $\eta-\eta^{\prime}$ system, see, e.g., J. Schechter, A. Subbaraman, and H. Weigel, Phys. Rev. D 48, (1993) 339.

14. S. Okubo, Phys. Rev. D 16, (1977) 2336; J. Jousset et al., (DM2 Collaboration), Phys. Rev. D 41, (1990) 1389 ; N. Morisita, I. Kitamura and T. Teshima, Phys. Rev. D 44, (1991) 175; R. Escribano, arXiv:hep-ph/0807.4201.

15. S. L. Glashow, Phys. Rev. Lett. 7, (1961) 469 .

16. S. A. Coon and R. C. Barrett, Phys. Rev. C 36, (1987) 2189 ; S. A. Coon, M. D. Scadron and P. C. McNamee, Nucl. Phys. A 287, (1977) 381; P. C. McNamee, M. D. Scadron and S. A. Coon, Nucl. Phys. A 249, (1975) 483; G. Krein, A. W. Thomas and A. G. Williams, Phys. Lett. B 317, (1993) 293; K. L. Mitchell, P.C. Tandy, C. D. Roberts and R. T. Cahill, Phys. Lett. B 335,(1994) 282 ; R. Machleidt and H. Muther, Phys. Rev. C 63, (2001) 034005 .

17. R. Enomoto and M. Tanabashi, Phys. Lett. B 386, (1996) 413.

18. S. Gardner, H. B. O'Connell and A. W. Thomas, Phys. Rev. Lett. 80, (1998) 1834.

19. X.-H. Guo and Z.-H. Zhang, Phys. Rev. D75,(2007) 074028

20. X.-H. Guo and A. W. Thomas, Phys. Rev. D61, (2000) 116009 .

21. K. Maltman and T. Goldman, Nucl. Phys. A 572, (1994) 682 .

22. J. Piekarewicz, Phys. Rev. C 48,(1993) 1555 ; T. Hatsuda, E.M. Henley, T. Meissner and G. Krein, Phys. Rev. C 49, (1994) 452 ; M.J. Iqbal and J.A. Niskanen, Phys. Lett. B 322,(1994) 7 ; H. B. O'Connell, B.C. Pearce, A. W. Thomas and A. G. Williams, Phys. Lett. B336, (1994)1 ; A. N. Mitra and K.C. Yang, Phys. Rev. C51, (1995) 3404 ; T. D. Cohen and G. A. Miller, Phys. Rev. C52, (1995) 3428 .

23. M. Benayoun and H. B. O'Connell, Eur. Phys. J. C 22, (2001) 503.

24. M. Benayoun, P. David, L. DelBuono, O. Leitner and H. B. O'Connell, Eur. Phys. J. C 55, (2008) 199.

25. M. Benayoun, arXiv:hep-ph/0805.1835.

26. D. Gusbin, Phys. Rev. D 24, (1981)797.

27. G. P. Lepage and S. J. Brodsky, Phys. Rev. D 22, (1980) 2157.

28. S. J. Brodsky, T. Huang, G. P. Lepage, in Quarks and Nuclear Forces, edited by D. Fries and B. Zeitnitz (Springer, Tracts in Modern Physics, Vol. 100) (Springer, New York, 1982); S. J. Brodsky, T. Huang and G. P. Lepage, in Particles and Fields-2, edited by A. Z. Capri and A. N. Kamal (Plenum, New York, 1983), p.143.

29. S. J. Brodsky, H.-C. Pauli and S. S. Pinsky, Phys. Rep. 301, (1998) 299.

30. H.-M. Choi and C.-R. Ji, Phys. Rev. D 56, (1997) 6010.

31. J. Cao, F.-G. Cao, T. Huang and B.-Q. Ma, Phys. Rev. D58, (1998) 113006.

32. H.-M. Choi and C.-R. Ji, Phys. Rev. D59, (1999) 074015. 
33. B.-W. Xiao and B.-Q. Ma, Phys. Rev. D 68, 034020 (2003);

Phys. Rev. D71, (2005) 014034.

34. T. Huang and X.-G. Wu, Eur. Phys. J. C 50, (2007) 771.

35. H. J. Melosh, Phys. Rev. D 9, (1974) 1095; E. Wigner, Ann. Math. 40, (1939) 149.

36. L. A. Kondratyuk and M. V. Terentev, Sov. J. Nucl. Phys. 31, (1980) 561 [Yad. Fiz. 31, (1980) 1087].

37. B.-Q. Ma, J. Phys. G 17, (1991) L53 [arXiv:hepph/0711.2335]; B.-Q. Ma, Q.-R. Zhang, Z. Phys. C 58, (1993) 479; B.-Q. Ma, Z. Phys. A 345, (1993) 321.

38. J. F. Donoghue, B. R. Holstein and Y. C. R. Lin, Phys. Rev. Lett. 55, (1985) 2766.

39. F. J. Gilman and R. Kauffman, Phys. Rev. D 36, (1987) 2761.

40. Th. Feldmann, P. Kroll and B. Stech, Phys. Lett. B 449, (1999) 339.

41. F.-G. Cao and A. I. Signal, Phys. Rev. D 60, (1999) 114012 .

42. W. Qian and B. -Q. Ma, Phys. Rev. D 78, (2008) 074002.

43. M. Benayoun, L. DelBuono, S. Eidelman, V. N. Ivanchenko and H. B. O'Connell, Phys. Rev. D 59, (1999) 114027.

44. G. M. Huber et al., (Jefferson Lab $F_{\pi}$ Collaboration), Phys. Rev. C 78, (2008) 045203.

45. C. J. Bebek, C. N. Brown and S. D. Holmes et al., Phys. Rev. D 17, (1978) 1693.

46. H.-J. Behrend et al., (CELLO Collaboration), Z. Phys. C 49, (1991) 401.

47. J. Gronberg et al., (CLEO Collaboration), Phys. Rev. D 57, (1998) 33 .

48. M. Acciarri et al., (L3 Collaboration), Phys. Lett. B 418, (1998) 399

49. T. Feldmann, Int. J. Mod. Phy. A 15, (2000) 159.

50. R. Escribano, and J. Frere, JHEP 06, (2005) 029.

51. T. Huang, B.-Q. Ma and Q.-X. Shen, Phys. Rev. D 49, (1994) 1490.

52. W. Jaus, Phys. Rev. D 44, (1991) 2851.

53. H.-M. Choi and C.-R. Ji, Nucl. Phy. A 618, (1997) 291.

54. C. Amsler, et al. (Particle Data Group), Phys. Lett. B 667, (2008) 1.

55. J.-H. Yu, B.-W. Xiao and B.-Q. Ma, J. Phys. G 34, (2007) 1845.

56. H.-M. Choi and C.-R. Ji, Nucl. Phys. A 679, (2001) 735.

57. R. Arnaldi et al. (NA60 Collaboration), arXiv:hep$\mathrm{ph} / 0902.2547$.

58. L.G. Landsberg, Phys. Rep. 128, (1985) 301.

59. A. N. Achasov et al., Phys. Lett. B 504, (2001) 275. 